Major article

\title{
Burden of present-on-admission infections and health care-associated infections, by race and ethnicity
}

\author{
Christie Y. Jeon $\mathrm{ScD}^{\mathrm{a}, *}$, Peter Muennig $\mathrm{PhD}^{\mathrm{b}}$, E. Yoko Furuya MD, MS ${ }^{\mathrm{c}}$, \\ Bevin Cohen $\mathrm{MPH}^{\mathrm{d}}$, Denis Nash $\mathrm{PhD}^{\mathrm{e}}$, Elaine L. Larson PhD, $\mathrm{RN}^{\mathrm{d}}$ \\ ${ }^{a}$ Department of Medicine, Cedars-Sinai Medical Center, Los Angeles, CA \\ ${ }^{\mathrm{b}}$ Department of Health Policy and Management, Mailman School of Public Health, Columbia University, New York, NY \\ ${ }^{\mathrm{c}}$ Division of Infectious Diseases, Department of Medicine, Columbia University, New York, NY \\ ${ }^{\mathrm{d}}$ School of Nursing, City University of New York School of Public Health, Hunter College Campus, New York, NY \\ ${ }^{\mathrm{e}}$ CUNY School of Public Health at Hunter College, Hunter College Campus, New York, NY
}

Key Words:

Health inequality

Community-acquired infections

Ambulatory care

\begin{abstract}
Background: In the United States incidence of sepsis and pneumonia differ by race, but it is unclear whether this is due to intrinsic factors or health care factors.

Methods: We conducted a study of 52,006 patients hospitalized during 2006-2008 at a referral hospital in upper Manhattan. We examined how the prevalence of present-on-admission and health care-associated infection compared between non-Hispanic blacks, Hispanics, and non-Hispanic whites adjusting for sociodemographic factors, admission through the emergency department, and comorbid conditions.

Results: Non-Hispanic blacks had 1.59-fold (95\% confidence interval [CI], 1.29-1.96) and 1.55-fold (95\% CI, 1.35-1.77) risk of community-acquired bloodstream infection and urinary tract infection compared with non-Hispanic whites. Hispanic patients had 1.31-fold (95\% CI, 1.15-1.49) risk of presenting with community-acquired urinary tract infection compared with non-Hispanic whites. Controlling for admission through the emergency department, comorbidity, and neighborhood income attenuated the differences in prevalence of infections.

Conclusions: We found that health disparities in present-on-admission infections might be largely explained by potential lack of ambulatory care, socioeconomic factors, and comorbidity.

Copyright $\odot 2014$ by the Association for Professionals in Infection Control and Epidemiology, Inc.
\end{abstract} Published by Elsevier Inc. All rights reserved.
In the United States incidence of sepsis and pneumonia differ by race, with blacks experiencing 1.25-2.4 times higher infection rates compared with whites. ${ }^{1-4}$ Understanding the multifactorial causes of this disparity and its influence on the source and types of infections would better inform how to reduce the excess burden of infection. Part of the disparity in infections could be attributed to differences in the prevalence of preexisting comorbidities. ${ }^{2,5}$ For example, blacks are more likely to develop sepsis because of underlying infection burden as well as organ dysfunction. ${ }^{4}$ Vulnerability to chronic conditions are affected by repeated exposure to

\footnotetext{
* Address correspondence to Christie Y. Jeon, ScD, Department of Medicine, Cedars-Sinai Medical Center, 8700 Beverly Blvd, Los Angeles, CA 90048.

E-mail address: christie.jeon@cshs.org (C.Y. Jeon).

To assure the integrity of our review process, the blinded peer review and final decision regarding publication for any manuscript on which the AJIC editor or an associate editor is an author is handled independently; that editor/associate editor has no decision making role in such manuscripts.

Conflicts of interest: None to report.
}

stressors ${ }^{6}$ as well as quality and access to care for these comorbidities $^{7}$ that are influenced by the larger socioeconomic context. However socioeconomic factors are only partly to be blamed because racial disparities in bacteremic pneumonia persisted even after adjustment for sociodemographic factors, including poverty level. ${ }^{1}$ Still others point to genetic differences in immune function for variability in predisposition to infections. ${ }^{8}$

Although previous studies have reported high rates of sepsis in blacks and highlight a few important mechanisms by which the disparity may occur, they did not simultaneously consider the influence of sociodemographic, comorbid, and health care-associated factors. Furthermore, there has been no attempt to distinguish whether the infections occurred in the community or in a health care setting. Differences in present-on-admission infection rates would call for better community-based prevention efforts, prevention through ambulatory care, culturally sensitive interventions, and lifestyle modifications, whereas differences in health careassociated infections would call for better management of 
inpatients in hospital settings. Previous studies on infection rates by race and ethnicity also lacked data on Hispanics, ${ }^{1,2,4}$ who now comprise the largest minority ethnic group in the United States.

In our study we compared rates of present-on-admission and health care-associated infections and described related demographic, clinical, and procedural factors by race and ethnicity, including Hispanics. Then we assessed if any apparent disparities in community-acquired infections are explained by referral through an emergency department and other factors present on admission. Further, we examined if any apparent disparity in health careassociated infections are explained by present-on-admission and in-hospital factors.

\section{METHODS}

\section{Study setting and patient population}

We conducted a retrospective study of patients who were discharged from January 2006-December 2008 from a tertiary referral hospital in upper Manhattan. We extracted data from the Clinical Data Warehouse that integrates information from more than 20 clinical electronic sources; the admission, discharge, and transfer system; and the computerized physician and nursing order entry system. Detailed description of the methods for integration of data have previously been published. ${ }^{9}$

\section{Case definition}

We examined racial and ethnic differences in 3 common types of infections: bloodstream infection (BSI), urinary tract infection (UTI), and pneumonia. Patients who develop an infection within 48 hours of admission are considered to have acquired it before admission. ${ }^{10}$ Because 48 hours could span over 3 days, we defined an infection for which the culture was collected within the first 3 days of admission as community-acquired, whereas infections that occurred thereafter were defined as health care-associated. We excluded people who had been hospitalized within 7 days before admission and those who transferred from other medical facilities, because they likely would not have community-acquired infection and the timing of infection from admission to a medical facility to infection onset could not be established. We followed infection algorithms recommended by the Centers for Disease Control and Prevention National Healthcare Safety Network ${ }^{10}$ and modified the definitions where clinical symptoms were indicated, because data on symptoms were not systematically collected in the electronic medical record system.

BSI was defined as infection that were confirmed with a positive blood culture for any bacterial pathogen and that had no positive culture with the same organism at other body sites within 14 days before positive blood culture. An infection with a common skin contaminant (eg, coagulase negative staphylococci) was counted as a case if 2 or more blood cultures drawn on separate occasions within 2 days of each other were positive.

UTI was defined as infections presenting with a positive urine culture as follows either $>10^{5} \mathrm{CFU} / \mathrm{mL}$ urine and no more than 1 other species of microorganism or $10^{3}-10^{5} \mathrm{CFU} / \mathrm{mL}$ urine and no more than 1 other species of microorganism, accompanied by pyuria within 2 days of positive culture.

We defined pneumonia as cases with 1 or more positive respiratory cultures and a discharge diagnosis of pneumonia, which includes a set of 62 ICD-9 codes indicative of pneumonia. Time to infection was determined by the day of culture collection for each type of infection.
Demographic, clinical, and procedural data

We obtained information on potential confounders, including age, sex, race/ethnicity, ICD-9-CM diagnoses, ICD-9-CM procedures, intensive care unit stay, admission through an emergency department, primary payer status, month and year of discharge, length of stay, and mortality from the ADT system. Race and ethnicity were categorized as non-Hispanic white, non-Hispanic black, Hispanic, other, or missing. Patients categorized as other included those who were identified in the system as "other (not specified)," "Asian," "Pacific Islander," "Indian," "Native Indian," or "Multi-racial." ICD-9$\mathrm{CM}$ diagnoses of interest included diabetes, renal failure, malignancy, transplant history, substance abuse, and chronic dermatitis that were present on admission. We computed the Charlson index of comorbidity, which has been validated to predict 10 -year mortality with each unit of increase in score, using ICD-9-CM codes to determine the extent of illness evident at admission. ${ }^{11}$ Procedures associated with infections included mechanical ventilation, vascular procedures (eg, cardiac catheterization, angiography, angioplasty, and stent), central venous catheterization, and urinary catheterization. Duration of central venous and urinary catheterization was determined from information available in the physicians' order sheets. Primary payers of hospital charges were categorized as Medicaid or non-Medicaid among those who were younger than age 65 years, and categorized as Medicare only, Medicare and supplemental insurance, or no Medicare among those aged 65 years or older. Socioeconomic status was determined by neighborhood median household income based on zip codelevel data from the 2000 US Census.

\section{Statistical analysis}

We described the characteristics of non-Hispanic white, nonHispanic black, and Hispanic patients, as well as those of other race and those missing racial identity by summarizing the frequencies and percentage of categorical variables and the mean $\pm S D$ of continuous variables. Differences in continuous variables by race and ethnicity categories were tested by Kruskal-Wallis test and differences in categorical variables were tested by $\chi^{2}$ tests. We examined the association between race and ethnicity and community-acquired infections via logistic regression, adjusting for age, sex, distance to the hospital, and year and month of discharge. Age, sex, distance to the hospital, and year and month of discharge were specified as confounders a priori because we were interested in contributors to disparity present, independent of these factors. Odds ratios (ORs) were interpreted as relative risks because the occurrence of the outcomes was $<5 \%$. Where a statistically significant difference in infection prevalence by race/ethnicity was detected, we examined potential contributors to the disparity by evaluating the association between race and ethnicity and community-acquired infections further adjusting for median neighborhood household income, primary payer, present-on-admission comorbid factors, and admission through an emergency department. Present-onadmission comorbid factors included diabetes, renal failure, malignancy, transplant history, substance abuse, chronic dermatitis, and Charlson score. The change in regression coefficients (log of OR) were used to determine how much variability in communityacquired infection by race/ethnicity could be explained by the above-mentioned factors.

We examined the association of race and ethnicity with health care-associated infections independent of age, sex, distance to the hospital, and year and month of discharge by Cox proportional hazard model, using days since admission as the scale of time. Where a statistically significant difference in hazard of infection was detected, we examined potential contributors to the disparity 
by evaluating the association between race and ethnicity and health care-associated infections adjusting for median household income, primary payer, comorbid factors, admission through an emergency department, and indwelling devices (ie, central venous line, vascular catheter, urinary catheter, and mechanical ventilation). To test for proportionality of hazards, we tested for interaction of log-transformed time with race/ethnicity and all other potential confounders and included significant interaction terms in the multivariate model. The percent change in regression coefficients (log of hazard ratio [HR]) was used to determine how much variability in community-acquired infection by race/ethnicity could be explained by the above-mentioned factors. Statistically significant associations were evaluated at $P \leq .05$ and all analyses were conducted in SAS version 9.2 (SAS Institute, Cary, NC).

\section{RESULTS}

\section{Study population}

There were 60,994 patients seen in the adult tertiary care hospital January 2006-December of 2008. After excluding 748 patients lacking neighborhood household income data and 8,345 patients who had transferred from other medical facilities or were previously hospitalized within 7 days, the final analytic sample was 52,006 patients. Of these, non-Hispanic whites, non-Hispanic blacks, Hispanics, other races, and those with unspecified race comprised $42 \%, 18 \%, 25 \%, 5.9 \%$, and $9.4 \%$ of the study population, respectively. The electronic medical records lacked data on the specific ethnicities of Hispanics; in 2007, Puerto Ricans and Dominicans comprised the largest ethnic groups among Hispanics in New York City. ${ }^{12}$ The non-Hispanic whites were older than all other categories of race/ethnicity (mean age, 60 vs 55 vs 55 vs 56 vs 55 years; $P<.0001$ ). Half of the study population was male, but this sex distribution varied by race-ethnic status, with a higher proportion of men among non-Hispanic whites compared with all others $(P<.0001)$.

Non-Hispanic blacks and Hispanics (relative to non-Hispanic whites) lived closer to the hospital $(P<.0001)$; came from a lower median neighborhood household income $(P<.0001)$; were more likely to have Medicaid membership $(P<.0001)$; and were more likely to be seen in an emergency room first $(P<.0001)$, have higher prevalence of diabetes and renal failure $(P<.0001)$, lower prevalence of transplant history and malignancy $(P<.0001$ for both), fewer operations $(P<.0001)$, and lower rates of certain invasive procedures $(P<.0001$ for central venous line, urinary catheter, and vascular catheter). See Table 1.

\section{Associations of race and ethnicity with community-acquired infections}

In our study population, $816,2,235$, and 280 patients presented with community-acquired BSIs, UTIs, and pneumonia, respectively, at admission. Adjusting for age, sex, distance to the hospital, and month and year of discharge non-Hispanic blacks had 1.59-fold higher risk of presenting with community-acquired BSI and 1.55fold higher risk of presenting with community-acquired UTI compared with non-Hispanic whites. Hispanic patients had 1.31fold risk of presenting with community-acquired UTIs compared with non-Hispanic whites. There was no statistically significant difference in prevalence of pneumonia by race and ethnic categories (Table 2). Other factors associated with a higher likelihood of community-acquired BSI included median neighborhood income $\leq \$ 75,000$, diabetes, renal failure, malignancy, transplant history, chronic dermatitis, higher Charlson score, and admission through the emergency department. Similarly, factors associated with a higher likelihood of community-acquired UTI included median neighborhood income $\leq \$ 75,000$, membership in Medicaid or Medicare Part A only, diabetes, renal failure, transplant history, chronic dermatitis, higher Charlson score, and admission through the emergency department (Table 2).

Controlling for other present-on-admission factors attenuated the differences in prevalence of infections to varying degrees (Table 3). Of note, adjusting for admission through the emergency department led to the greatest attenuation in the relationship between race/ethnicity and community acquired BSIs and UTIs to null associations. The second highest contributing factor was comorbidity (including diabetes, renal failure, malignancy, transplant history, substance abuse, chronic dermatitis, and Charlson score), which collectively explained $26 \%-42 \%$ of the disparity between non-Hispanic blacks and non-Hispanic whites and $19 \%$ of the disparity between Hispanics and non-Hispanic whites. Median neighborhood household income also contributed substantially accounting for $20 \%-27 \%$ of the apparent disparity (Table 3 ).

Associations of race and ethnicity with health care-associated infections

Of those who were admitted to the hospital without communityacquired infection and stayed in the hospital for longer than 3 days, $506,1,026$, and 4,284 patients developed health care-associated BSI, UTI, and pneumonia, respectively. Adjusting for age, sex, distance to the hospital, and month and year of discharge, an elevated hazard of health care-associated BSI was apparent among non-Hispanic blacks compared with non-Hispanic whites (HR, 1.31; 95\% confidence interval [CI],1.02-1.69). When modeling the hazard of UTI, the interaction between time and race-ethnicity was significant. The estimated HR at baseline was $>1$, similar to trends observed in community-acquired UTI (Table 2). However, the ratio estimated for the interaction term with time was $<1$, which indicates that the difference in occurrence of UTI by race categories that was evident at admission (time $=0$ ) decreased with increasing time. There was no statistically significant difference in hazard of pneumonia by race and ethnic categories (Table 4). Other factors associated with health care-associated BSIs include Medicaid membership, renal failure, malignancy, higher Charlson index, admission through an emergency department, urinary catheterization, and central venous line catheterization.

Controlling for admission through the emergency department, primary payer and comorbidity attenuated the differences in hazard of infections between non-Hispanic blacks and whites by $17 \%$, $15 \%$, and $13 \%$, respectively (Table 5 ). Controlling for indwelling devices did not account for the difference, but rather further strengthened the relationship between non-Hispanic black ethnicity and health care-associated infection (ie, increased the magnitude of coefficient). When all variables were adjusted for, non-Hispanic black patients were not at significantly higher risk for health care-associated BSI (HR, $1.14^{0.135} ; 95 \% \mathrm{CI}, 0.87-1.51$ ) (Table 5).

\section{Associations of race and ethnicity with admission through an emergency department}

To determine factors that influenced racially disparate patterns of admission through the emergency department, we investigated how controlling for primary payer, median neighborhood household income, and comorbid factors changed the coefficient of association between race/ethnicity and admission through the emergency department. Adjusting for age, sex, distance to the hospital, and month and year of discharge, non-Hispanic blacks and Hispanics were more likely to be hospitalized through the emergency department compared with non-Hispanic whites (OR, 3.92; 
Table 1

Description of demographic and clinical characteristics by race and ethnicity in a tertiary referral hospital for adults

\begin{tabular}{|c|c|c|c|c|c|}
\hline Categorical variable & $\begin{array}{c}\text { Non-Hispanic white } \\
\quad(\mathrm{n}=24,045)\end{array}$ & $\begin{array}{l}\text { Non-Hispanic black } \\
\qquad(\mathrm{n}=9210)\end{array}$ & $\begin{array}{c}\text { Hispanic } \\
(\mathrm{n}=12,795)\end{array}$ & $\begin{array}{c}\text { Other } \\
(\mathrm{n}=3070)\end{array}$ & $\begin{array}{c}\text { Missing } \\
(\mathrm{n}=4886)\end{array}$ \\
\hline \multicolumn{6}{|l|}{ Infection } \\
\hline \multicolumn{6}{|l|}{ Community-acquired Infection (within $3 \mathrm{~d}$ of admission) } \\
\hline BSI & $235(1.1)$ & $216(2.4)$ & $241(1.9)$ & $39(1.3)$ & $85(1.7)$ \\
\hline UTI & $611(2.8)$ & $560(6.1)$ & $711(5.6)$ & $105(3.4)$ & $248(5.1)$ \\
\hline Pneumonia & $98(0.4)$ & $70(0.8)$ & $72(0.6)$ & $9(0.3)$ & $31(0.6)$ \\
\hline \multicolumn{6}{|l|}{ Health care-associated infection } \\
\hline BSI (case/1,000 person $\mathrm{d}$ ) & $193(2.80)$ & $122(3.70)$ & $123(3.23)$ & $24(2.62)$ & $44(2.47)$ \\
\hline UTI (case/1,000 person d) & $431(6.57)$ & $202(6.63)$ & $229(6.38)$ & $56(6.68)$ & $108(6.39)$ \\
\hline Pneumonia (case/1,000 person $\mathrm{d}$ ) & $162(2.35)$ & $96(2.78)$ & $100(2.56)$ & $24(2.64)$ & $46(2.55)$ \\
\hline \multicolumn{6}{|l|}{ Factors present on admission } \\
\hline Age (mean $\mathrm{y} \pm \mathrm{SD}$ ) & $60 \pm 17$ & $55 \pm 17$ & $55 \pm 18$ & $56 \pm 17$ & $55 \pm 19$ \\
\hline Male & $12,094(55)$ & $4,167(45)$ & $5,661(45)$ & $1,590(52)$ & $2,519(52)$ \\
\hline Admission through the emergency department & $4,929(22)$ & $6,651(72)$ & $9,587(75)$ & $1,185(39)$ & $2,961(61)$ \\
\hline \multicolumn{6}{|l|}{ Distance to hospital (miles) } \\
\hline$<2$ & $1,936(8.8)$ & $4,439(48)$ & $7,352(57)$ & $630(21)$ & $1,683(34)$ \\
\hline $2-5$ & $2,323(11)$ & $2,155(23)$ & $3,233(25)$ & $438(14)$ & $788(16)$ \\
\hline$>5$ & $17,786(81)$ & $2,616(28)$ & $2,210(17)$ & $2,002(65)$ & $2,415(49)$ \\
\hline Neighborhood median household income (mean $\$ \pm S D$ ) & $60,836 \pm 26,528$ & $30,095 \pm 13,448$ & $30,447 \pm 12,185$ & $47,375 \pm 24,750$ & $43,882 \pm 25,510$ \\
\hline \multicolumn{6}{|l|}{ Primary payer } \\
\hline \multicolumn{6}{|l|}{ Age $<65$ y } \\
\hline Medicaid & $672(3.1)$ & $2,132(23)$ & $3,270(26)$ & $366(12)$ & $698(14)$ \\
\hline Private & $11,577(53)$ & $4,333(47)$ & $5,372(42)$ & $1,632(53)$ & $2,604(53)$ \\
\hline \multicolumn{6}{|l|}{ Age $\geq 65$ y } \\
\hline No Medicare & $1,108(5.0)$ & $464(5.0)$ & $847(6.6)$ & $200(6.5)$ & $263(5.4)$ \\
\hline Medicare Part A only & $7,723(35)$ & $1,661(18)$ & $2,581(20)$ & $672(22)$ & $1,034(21)$ \\
\hline Medicare Part A + other & $965(4.4)$ & $620(6.7)$ & $725(5.9)$ & $200(6.5)$ & $287(5.9)$ \\
\hline \multicolumn{6}{|l|}{ Comorbidity present on admission } \\
\hline Diabetes & $3,314(15)$ & $2,150(23)$ & $3,318(26)$ & $710(23)$ & $833(17)$ \\
\hline Renal failure & $2,138(10)$ & $1,628(18)$ & $1,608(13)$ & $375(12)$ & $547(11)$ \\
\hline Malignancy & $3,742(17)$ & $914(10)$ & $1,150(9.0)$ & $357(12)$ & $494(10)$ \\
\hline Transplant history & $550(2.5)$ & $131(1.4)$ & $213(1.7)$ & $48(1.6)$ & $35(0.7)$ \\
\hline Substance abuse & $629(2.9)$ & $1,103(12)$ & $828(6.5)$ & $74(2.4)$ & $377(7.7)$ \\
\hline Chronic dermatitis & $587(2.7)$ & $388(4.2)$ & $517(4.0)$ & $81(2.6)$ & $171(3.5)$ \\
\hline Charlson score* $2+$ & $7,034(32)$ & $3,358(36)$ & $3,960(31)$ & $990(32)$ & $1,349(28)$ \\
\hline \multicolumn{6}{|l|}{ During hospitalization } \\
\hline ICU stay & $2,560(12)$ & $752(8.2)$ & $1,033(8.0)$ & $316(10)$ & $448(9.2)$ \\
\hline Operations $>30 \mathrm{~min}$ & $10,585(48)$ & $1,989(22)$ & $2,903(23)$ & $947(31)$ & $1,281(26)$ \\
\hline Mechanical ventilation & $644(2.9)$ & $335(3.6)$ & $451(3.5)$ & $76(2.5)$ & $188(3.9)$ \\
\hline Vascular catheters & $4,827(22)$ & $1,118(12)$ & $1,600(13)$ & $941(31)$ & $644(13)$ \\
\hline Urinary catheter & $11,400(52)$ & $2,981(32)$ & $3,963(31)$ & $1,265(41)$ & $1,585(32)$ \\
\hline Central venous line & $2,361(11)$ & $598(6.5)$ & $692(5.4)$ & $282(9.2)$ & $286(5.9)$ \\
\hline
\end{tabular}

NOTE. Values are given as $\mathrm{n}(\%)$ unless otherwise noted.

$A D T$, admissions, discharge and transfer record; BSI, bloodstream infection; ICU, intensive care unit; UTI, urinary tract infection.

${ }^{*}$ Greater Charlson index score indicates greater presence of illness that increase the 10-year predicted mortality.

95\% CI, 3.67-4.18 and OR, 3.28; 95\% CI, 3.09-3.49). Collectively, neighborhood household income, primary payer, and comorbidity explained $35 \%$ of the excess emergency department visits among non-Hispanic blacks and 31\% that of Hispanics; of these, neighborhood household income accounted for $23 \%$ of the disparity in non-Hispanic blacks and $22 \%$ in Hispanics. After adjustment for all present-on-admission factors, Hispanic blacks and Hispanics were still more likely to be hospitalized through the emergency department compared with non-Hispanic whites (OR, 2.44; 95\% CI, 2.27-2.62 for non-Hispanic blacks and OR, 2.26; 95\% CI, 2.11-2.42 for Hispanics).

\section{DISCUSSION}

In this population of more than 50,000 inpatients, we found that non-Hispanic blacks were at slightly elevated risk of developing BSI throughout the hospital stay compared with non-Hispanic whites. Could this have been influenced by differential treatment of blacks in the hospitals? The data indicate that although non-Hispanic blacks were less likely to receive indwelling devices, this had no bearing on the difference in incidence of infections. This association was partially reduced by adjustment for admission through an emergency department, suggesting that the emergency department could be a source of health care-associated infection, as has been previously hypothesized. ${ }^{13}$ The data also suggest that the relative hazard of UTI by race and ethnicity decreased over time, indicating that differences apparent toward the beginning of the admission disappeared as patients spent more time in the hospital. This further suggests that in this hospital setting differences in infection rates are likely driven by factors present before admission.

In addition to our findings for health care-associated BSI, we found greater prevalence of community-acquired BSI and UTI in non-Hispanic blacks and Hispanics, compared with non-Hispanic whites after accounting for demographic and temporal factors. On the other hand we found no differences in the presence of community-acquired or health care-associated pneumonia by race or ethnicity. Previous studies on race and infection reported a similarly higher crude or age-adjusted rate of sepsis ${ }^{4,5}$ and overall infection rates ${ }^{4}$ in African Americans compared with whites. Our study adds to the literature by demonstrating that apparent disparities in community-associated BSI and UTI can be explained in part by multiple present-on-admission factors, including admission through an emergency department, comorbid factors, and socioeconomic context. We also contribute to the discussion of health inequity by including the Hispanic population, which is the fastest growing minority group in the United States. 
Table 2

Age-, sex-, distance to hospital-, and month- and year-adjusted associations with community-acquired infections

\begin{tabular}{|c|c|c|c|}
\hline & $\begin{array}{l}\text { Community-acquired } \\
\text { bloodstream infection }\end{array}$ & $\begin{array}{l}\text { Community-acquired } \\
\text { urinary tract infection }\end{array}$ & $\begin{array}{c}\text { Community-acquired } \\
\text { pneumonia }\end{array}$ \\
\hline \multicolumn{4}{|l|}{ Factors present on admission } \\
\hline \multicolumn{4}{|l|}{ Race/ethnicity (non-Hispanic white as reference) } \\
\hline Non-Hispanic black & $1.59(1.29-1.96)$ & $1.55(1.35-1.77)$ & $1.36(0.96-1.93)$ \\
\hline Hispanic & $1.19(0.96-1.47)$ & $1.31(1.15-1.49)$ & $0.96(0.67-1.37)$ \\
\hline Other & $1.10(0.78-1.55)$ & $1.14(0.92-1.42)$ & $0.62(0.31-1.23)$ \\
\hline Missing & $1.35(1.04-1.76)$ & $1.56(1.33-1.83)$ & $1.27(0.83-1.94)$ \\
\hline Age ( $1 \mathrm{y}$ increase in age) & $1.014(1.010-1.018)$ & $1.023(1.020-1.026)$ & $1.012(1.005-1.019)$ \\
\hline Male & $1.45(1.26-1.67)$ & $0.40(0.37-0.44)$ & $1.64(1.29-2.09)$ \\
\hline \multicolumn{4}{|l|}{ Distance to the hospital ( $\leq 2$ miles as reference) } \\
\hline 2-5 miles & $0.79(0.66-0.95)$ & $0.80(0.71-0.89)$ & $0.81(0.59-1.12)$ \\
\hline$>5$ miles & $0.35(0.30-0.42)$ & $0.37(0.33-0.40)$ & $0.49(0.37-0.63)$ \\
\hline \multicolumn{4}{|c|}{ Neighborhood median household income ( $>\$ 75,000$ as reference } \\
\hline$\$ 50,001-\$ 75,000$ & $1.70(1.20-2.43)$ & $1.32(1.07-1.63)$ & $1.16(0.70-1.92)$ \\
\hline$\$ 30,001-\$ 50,000$ & $2.00(1.42-2.81)$ & $1.59(1.30-1.94)$ & $1.34(0.82-2.18)$ \\
\hline$\leq \$ 30,000$ & $2.28(1.60-3.26)$ & $1.83(1.49-2.26)$ & $1.90(1.12-3.20)$ \\
\hline \multicolumn{4}{|l|}{ Primary payer } \\
\hline \multicolumn{4}{|l|}{ Age $<65$ y } \\
\hline \multicolumn{4}{|c|}{ Private health maintenance organization insurance as reference } \\
\hline Medicaid & $1.26(1.02-1.55)$ & $1.54(1.34-1.76)$ & $1.90(1.35-2.67)$ \\
\hline \multicolumn{4}{|l|}{ Age $\geq 65 y$} \\
\hline Medicare Part $\mathrm{A}+$ other & $0.95(0.68-1.34)$ & $1.18(0.96-1.46)$ & $1.12(0.63-1.99)$ \\
\hline Medicare Part A only & $1.01(0.78-1.31)$ & $1.39(1.18-1.64)$ & $0.98(0.63-1.53)$ \\
\hline No Medicare/private & $0.79(0.56-1.13)$ & $1.02(0.82-1.27)$ & $1.10(0.63-1.99)$ \\
\hline \multicolumn{4}{|l|}{ Comorbidity present on admission } \\
\hline Diabetes & $1.22(1.04-1.43)$ & $1.36(1.24-1.50)$ & $1.09(0.82-1.44)$ \\
\hline Renal failure & $4.48(3.86-5.21)$ & $2.63(2.37-2.91)$ & $3.43(2.65-4.45)$ \\
\hline Malignancy & $1.63(1.36-1.96)$ & $1.13(0.99-1.28)$ & $0.98(0.68-1.40)$ \\
\hline Transplant history & $2.92(2.07-4.12)$ & $2.52(1.96-3.25)$ & $2.10(1.11-3.97)$ \\
\hline Substance abuse & $1.14(0.86-1.51)$ & $0.98(0.79-1.22)$ & $2.40(1.64-3.50)$ \\
\hline Chronic dermatitis & $2.81(2.22-3.55)$ & $1.48(1.22-1.80)$ & $0.91(0.48-1.71)$ \\
\hline Charlson score $2+$ versus 0 or 1 & $2.63(2.27-3.05)$ & $1.58(1.45-1.73)$ & $2.66(2.08-3.42)$ \\
\hline Admission through an emergency department & $7.49(5.97-9.41)$ & $5.85(5.12-6.68)$ & $3.73(2.69-5.17)$ \\
\hline
\end{tabular}

NOTE. Values are given as odds ratio (95\% confidence interval).

Table 3

Contribution of present-on-admission infection on the difference in log odds of community-acquired infections by race/ethnicity

\begin{tabular}{|c|c|c|c|c|c|c|}
\hline \multirow[b]{2}{*}{ Model } & \multicolumn{2}{|c|}{ Community-acquired bloodstream infection } & \multicolumn{4}{|c|}{ Community-acquired urinary tract infection } \\
\hline & $\begin{array}{c}\text { Coefficient for } \\
\text { non-Hispanic } \\
\text { black versus white }\end{array}$ & \% Reduction & $\begin{array}{c}\text { Coefficient for } \\
\text { non-Hispanic } \\
\text { black versus white }\end{array}$ & \% Reduction & $\begin{array}{l}\text { Coefficient for } \\
\text { Hispanic } \\
\text { versus white }\end{array}$ & \% Reduction \\
\hline $\begin{array}{l}\text { Model A: Age, sex, distance to hospital, month, } \\
\text { and year adjusted }\end{array}$ & 0.463 & - & 0.436 & - & 0.267 & - \\
\hline $\begin{array}{l}\text { Model } \mathrm{A}+\text { admission through an emergency } \\
\text { department }\end{array}$ & 0.035 & 92 & 0.006 & 87 & -0.070 & 126 \\
\hline Model A + comorbidity & 0.270 & 42 & 0.321 & 26 & 0.215 & 19 \\
\hline $\begin{array}{l}\text { Model A + median neighborhood household } \\
\text { income }\end{array}$ & 0.358 & 23 & 0.351 & 20 & 0.197 & 27 \\
\hline Model A + primary payer status & 0.459 & 1 & 0.440 & $0^{*}$ & 0.259 & 3 \\
\hline All variables & -0.058 & 112 & 0.029 & 93 & -0.07 & 128 \\
\hline
\end{tabular}

*Negative \% value was replaced with $0 \%$ because the inclusion of the covariates explains greater difference than exists by race/ethnic categories.

Of potentially contributing factors, controlling for admission through the emergency department led to the greatest reduction in disparity in present-on-admission infections. Presentation to an emergency department could reflect a higher acuity of illness, lack of ambulatory or primary care options and health insurance, or norms surrounding appropriate sources of care (eg, distrust of the health care system or concerns about immigration status). These factors are intrinsically linked. Inadequate access to ambulatory care may result in hospitalizations with severe infections due to suboptimal management of underlying disease. Poorly managed chronic diseases, such as renal failure, increase the risk of infection, as well as delay diagnosis and treatment, during which infections progress to severe levels. Indeed, racial disparities in hospitalizations for ambulatory care-sensitive conditions, ${ }^{14}$ use of emergency department services, and time to filling prescription ${ }^{15}$ have been reported in the literature. However, these studies show that the differences persisted even after controlling for socioeconomic measures and insurance status. Disparate patterns of admission through emergency departments by race/ethnicity also remained in our study after controlling for socioeconomic status, indicating that other culture-level or community-level factors also influence racial disparity in use of emergency department services.

In models that did not include admission through an emergency department, we found that comorbidity and socioeconomic context explained a substantial burden of the disparity in communityacquired BSI and UTI. The specific comorbid conditions that were present to a greater extent in non-Hispanic blacks and Hispanic populations in our study were diabetes, renal failure, substance abuse, and chronic dermatitis, which are known risk factors for infection. ${ }^{16-19}$ Household income levels are correlated with 
Table 4

Age-, sex-, distance to hospital-, month-, and year-adjusted associations with health care-associated infections

\begin{tabular}{|c|c|c|c|}
\hline & $\begin{array}{l}\text { Health care-associated } \\
\text { bloodstream infection }\end{array}$ & $\begin{array}{l}\text { Health care-associated } \\
\text { urinary tract infection }\end{array}$ & $\begin{array}{l}\text { Health care-associated } \\
\text { pneumonia }\end{array}$ \\
\hline \multicolumn{4}{|l|}{ Factor present on admission } \\
\hline \multicolumn{4}{|l|}{ Race/ethnicity (non-Hispanic white as reference) } \\
\hline Non-Hispanic black & $1.31(1.02-1.69)$ & $1.19(0.98-1.46)^{*}$ & $1.14(0.86-1.51)$ \\
\hline Hispanic & $1.19(0.92-1.54)$ & $1.36(1.07-1.73)^{*}$ & $1.11(0.84-1.48)$ \\
\hline Other & $0.94(0.61-1.45)$ & $1.46(1.02-2.08)^{*}$ & $1.18(0.77-1.82)$ \\
\hline Missing & $0.79(0.56-1.10)$ & $2.02(1.08-3.76)^{*}$ & $1.10(0.79-1.55)$ \\
\hline Interaction with time* & $\mathrm{NA}^{\dagger}$ & $0.96(0.93-0.99)$ & $\mathrm{NA}^{\dagger}$ \\
\hline Age ( $1 \mathrm{y}$ increase in age) & $1.008(1.003-1.014)$ & $1.021(1.018-1.025)$ & $1.016(1.010-1.021)$ \\
\hline Male & $2.80(1.59-4.94)$ & $0.42(0.37-0.47)$ & $1.54(1.26-1.88)$ \\
\hline Interaction with time* & $0.72(0.58-0.90)$ & $\mathrm{NA}^{\dagger}$ & $\mathrm{NA}^{\dagger}$ \\
\hline \multicolumn{4}{|l|}{ Distance to the hospital ( $\leq 2$ miles as reference) } \\
\hline 2-5 miles & $0.84(0.65-1.09)$ & $0.91(0.75-1.09)$ & $1.18(0.91-1.54)$ \\
\hline$>5$ miles & $0.82(0.67-0.99)$ & $1.00(0.87-1.15)$ & $0.91(0.73-1.14)$ \\
\hline \multicolumn{4}{|c|}{ Neighborhood median household income ( $>\$ 75,000$ as reference) } \\
\hline$\$ 50,001-\$ 75,000$ & $0.87(0.63-1.21)$ & $0.83(0.67-1.03)$ & $1.45(1.08-2.26)$ \\
\hline$\$ 30,001-\$ 50,000$ & $0.88(0.65-1.21)$ & $0.87(0.71-1.08)$ & $1.23(0.85-1.78)$ \\
\hline$\leq \$ 30,000$ & $1.10(0.77-1.57)$ & $0.97(0.76-1.23)$ & $0.98(0.65-1.48)$ \\
\hline \multicolumn{4}{|l|}{ Primary payer } \\
\hline \multicolumn{4}{|c|}{ Age $<65$ y } \\
\hline \multicolumn{4}{|c|}{ Private health maintenance organization insurance as reference } \\
\hline Medicaid & $1.34(1.02-1.75)$ & $0.92(0.73-1.15)$ & $1.16(0.85-1.58)$ \\
\hline \multicolumn{4}{|l|}{ Age $\geq 65 \mathrm{y}$} \\
\hline Medicare Part $\mathrm{A}+$ other & $0.83(0.54-1.28)$ & $1.11(0.83-1.49)$ & $1.19(0.76-1.86)$ \\
\hline Medicare Part A only & $0.86(0.62-1.19)$ & $1.06(0.84-1.33)$ & $1.10(0.77-1.57)$ \\
\hline No Medicare/private & $0.85(0.54-1.33)$ & $1.01(0.74-1.39)$ & $0.89(0.55-1.44)$ \\
\hline \multicolumn{4}{|l|}{ Comorbidity present on admission } \\
\hline Diabetes & $1.03(0.84-1.27)$ & $1.10(0.95-1.27)$ & $1.18(0.95-1.46)$ \\
\hline Renal failure & $1.36(1.13-1.64)$ & $0.90(0.78-1.05)$ & $1.35(1.12-1.62)$ \\
\hline Malignancy & $1.38(1.13-1.69)$ & $1.23(1.06-1.41)$ & $1.01(0.82-1.25)$ \\
\hline Transplant history & $1.45(0.92-2.26)$ & $0.98(0.61-1.54)$ & $1.01(0.60-1.70)$ \\
\hline Substance abuse & $0.69(0.47-1.02)$ & $0.87(0.64-1.17)$ & $0.74(0.49-1.12)$ \\
\hline Chronic dermatitis & $0.83(0.58-1.19)$ & $0.59(0.44-0.80)$ & $0.89(0.64-1.24)$ \\
\hline Charlson score $2+$ versus 0 or 1 & $1.44(1.19-1.74)$ & $1.22(1.07-1.38)$ & $1.24(1.03-1.50)$ \\
\hline Admission through an emergency department & $1.29(1.03-1.61)$ & $0.31(0.21-0.46)$ & $1.14(0.92-1.40)$ \\
\hline Interaction with time & NA & $1.55(1.31-1.82)$ & $\mathrm{NA}^{\dagger}$ \\
\hline \multicolumn{4}{|l|}{ During the hospitalization } \\
\hline Intensive care unit stay & $1.02(0.85-1.23)$ & $0.87(0.77-1.00)$ & $6.12(4.89-7.65)$ \\
\hline Operations $>30 \mathrm{~min}$ & $0.88(0.72-1.07)$ & $1.17(1.02-1.33)$ & $1.29(1.07-1.56)$ \\
\hline Mechanical ventilation & $0.95(0.77-1.17)$ & $0.04(0.02-0.08)$ & $5.71(4.76-6.86)$ \\
\hline Interaction with time* & $\mathrm{NA}^{\dagger}$ & $2.89(2.25-3.71)$ & $\mathrm{NA}^{\dagger}$ \\
\hline Vascular catheters & $0.23(0.10-0.53)$ & $0.92(0.78-1.09)$ & $1.01(0.80-1.27)$ \\
\hline Interaction with time* & $1.62(1.21-2.17)$ & $\mathrm{NA}^{\dagger}$ & $\mathrm{NA}^{\dagger}$ \\
\hline Urinary catheter & $1.37(1.15-1.65)$ & $2.14(1.88-2.43)$ & $2.47(2.04-3.00)$ \\
\hline Central venous line & $1.48(1.21-1.82)$ & $0.58(0.49-0.70)$ & $4.79(3.97-5.78)$ \\
\hline
\end{tabular}

NOTE. Values are given as hazard ratio (95\% confidence interval).

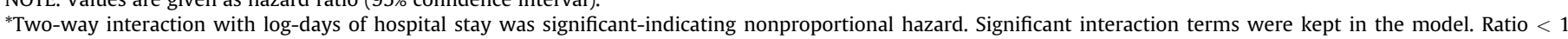

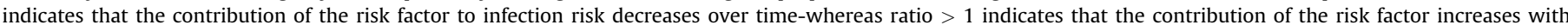

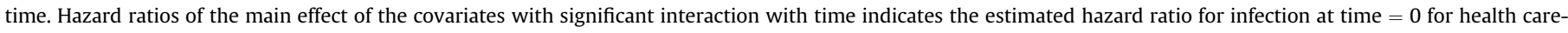
associated infections (day 4 of admission) based on changing relative hazard over time.

'Interaction with log-days of hospital stay was not significant and therefore not included in the model.

measures of crowding ${ }^{20}$ that could elevate the exposure to and transmission of infectious agents. Disparities unexplained by measured comorbidity and socioeconomic context may also be influenced by differences in allostatic load, which affects the neuroendocrine and immune systems. ${ }^{6,21}$ Allostatic load leads to a resetting of the body's natural feedback systems, which may result in an increased risk of chronic disease as well as an altered response (and susceptibility to) immunologic threats.

The strengths of our study include a large sample of a hospitalized adult population, with sufficient representation of nonHispanic blacks and Hispanics to be able to have $80 \%$ power to detect $>20 \%$ difference between the racial groups for the most common infection (ie, community-acquired UTI). The collection of data from multiple sources of electronic records allowed for control of multiple potential confounders, including demographic characteristics, insurance status, comorbid conditions, and in-hospital variables. The database also allowed us to distinguish between community-acquired and health care-associated infections and establishing the temporal order of administration of invasive procedures and infection events.

The use of existing electronic data also posed some limitations. First, we did not have access to systematic data on symptoms of infections, which could have led to underreporting of cases. The use of laboratory culture data allowed for identification of bacterial infections specifically and enabled us to determine when the infection was suspected. Furthermore, it is unlikely that culture collection would have been influenced by race or ethnicity. We lacked data on health behaviors, housing conditions, and individual income levels that could collectively influence one's risk of community-acquired infection. In this regard, the fact that blatant disparities in infection disappeared with the adjustment of variables suggests that the measured confounding variables accounted 
Table 5

Contribution of present-on-admission and in-hospital factors on the difference in log-hazard of health care-associated bloodstream, by race/ethnicity

\begin{tabular}{llc}
\hline & \multicolumn{2}{c}{$\begin{array}{c}\text { Health care-associated } \\
\text { bloodstream infection }\end{array}$} \\
\cline { 2 - 3 } & $\begin{array}{c}\text { Coefficient for } \\
\text { non-Hispanic } \\
\text { black versus white }\end{array}$ & \% Reduction \\
\hline Model & 0.270 & - \\
\hline Model A: Age, sex, distance to hospital, & & 17 \\
$\quad$ month, and year adjusted & 0.223 & 15 \\
$\quad$ Model A + Admission through the & & 13 \\
Model A + primary payer status & 0.231 & 5 \\
Model A + comorbidity & 0.235 & 3 \\
Model A + median neighborhood & 0.257 & $0^{*}$ \\
$\quad$ household income & & 50 \\
Model A + central venous line & 0.263 & 0.275 \\
Model A + other invasive procedures & 0.135 & \\
All variables & &
\end{tabular}

*Negative \% value was replaced with $0 \%$ because the inclusion of the covariates explains greater difference than exists by race/ethnic categories.

for differences in unmeasured positive confounders. Because we had access to data from only hospitalized patients, our findings on community-acquired infection may not be generalizable to community-acquired infection events that do not require hospitalization. The absence of data on individuals' access to ambulatory care services also prevented us from determining whether the differential referral through the emergency department was due to lack of ambulatory options. Finally, as mentioned earlier, data on other racial or ethnic groups were relatively sparse and the specific ethnic identities of patients in this category were unclear.

We found greater prevalence of community-acquired BSI and UTI in hospitalized non-Hispanic blacks and Hispanics compared with non-Hispanic whites. The differences were largely explained by multiple present-on-admission factors, admission through the emergency department, neighborhood income levels, and comorbid conditions. We also found higher incidence of health careassociated BSI in Hispanics that was attenuated with control for admission through an emergency department. The greater use of emergency departments among non-Hispanic blacks and Hispanics could only be partially explained by socioeconomic context, primary payer, and comorbid conditions.

\section{Future directions}

Our study points to the research needs for determining how to prevent excess hospitalizations with infections among minorities through enhanced ambulatory care. This study also points to the need for community-level policies that might enhance health and reduce health disparities. Intervention studies to improve health outcomes in the community have examined a number of social goods, such as income tax reduction, ${ }^{22}$ enhanced education, ${ }^{23-25}$ housing vouchers, ${ }^{26,27}$ and conditional cash transfers. ${ }^{28-34}$ However these studies have not produced consistent results on what social interventions are effective in improving health outcomes. Therefore, not only are more intervention studies on the nonmedical determinants of health needed, but also new ways of effectively addressing differences in access to social goods in different racial and ethnic contexts.

\section{References}

1. Burton DC, Flannery B, Bennett NM, Farley MM, Gershman K, Harrison LH, et al. Socioeconomic and racial/ethnic disparities in the incidence of bacteremic pneumonia among US adults. Am J Public Health 2010;100:1904-11.
2. Esper AM, Moss M, Lewis CA, Nisbet R, Mannino DM, Martin GS. The role of infection and comorbidity: factors that influence disparities in sepsis. Crit Care Med 2006;34:2576-82.

3. Martin GS, Mannino DM, Eaton S, Moss M. The epidemiology of sepsis in the United States from 1979 through 2000. N Engl J Med 2003;348:1546-54.

4. Mayr FB, Yende S, Linde-Zwirble WT, Peck-Palmer OM, Barnato AE, Weissfeld LA, et al. Infection rate and acute organ dysfunction risk as explanations for racial differences in severe sepsis. JAMA 2010;303:2495-503.

5. Dombrovskiy VY, Martin AA, Sunderram J, Paz HL. Occurrence and outcomes of sepsis: influence of race. Crit Care Med 2007;35:763-8.

6. Geronimus AT, Hicken M, Keene D, Bound J. "Weathering" and age patterns of allostatic load scores among blacks and whites in the United States. Am J Public Health 2006;96:826-33.

7. Nelson A. Unequal treatment: confronting racial and ethnic disparities in health care. J Natl Med Assoc 2002;94:666-8.

8. Ferwerda B, McCall MB, Alonso S, Giamarellos-Bourboulis EJ, Mouktaroudi M, Izagirre $\mathrm{N}$, et al. TLR4 polymorphisms, infectious diseases, and evolutionary pressure during migration of modern humans. Proc Natl Acad Sci U S A 2007; 104:16645-50.

9. Apte M, Neidell M, Furuya EY, Caplan D, Glied S, Larson E. Using electronically available inpatient hospital data for research. Clin Transl Sci 2011;4:338-45.

10. Horan TC, Andrus M, Dudeck MA. CDC/NHSN surveillance definition of health care-associated infection and criteria for specific types of infections in the acute care setting. Am J Infect Control 2008;36:309-32.

11. Valderas JM, Starfield B, Sibbald B, Salisbury C, Roland M. Defining comorbidity: implications for understanding health and health services. Ann Fam Med 2009; 7:357-63.

12. Limonic L. The Latino Population of New York City, 2007. New York: Center for Latin American, Caribbean and Latino Studies, City University of New York; 2008.

13. Quach C, McArthur M, McGeer A, Li L, Simor A, Dionne M, et al. Risk of infection following a visit to the emergency department: a cohort study. CMAJ 2012; 184:E232-9.

14. O’Neil SS, Lake T, Merrill A, Wilson A, Mann DA, Bartnyska LM. Racial disparities in hospitalizations for ambulatory care-sensitive conditions. Am J Prev Med 2010;38:381-8.

15. Roby DH, Nicholson GL, Kominski GF. African Americans in commercial HMOs more likely to delay prescription drugs and use the emergency room. Policy Brief UCLA Cent Health Policy Res; 2009:1-12. PB2009-7.

16. Frykberg RG. An evidence-based approach to diabetic foot infections. Am J Surg 2003:186:44S-54S. discussion 61S-4S.

17. Bennett JV, Jarvis WR, Brachman PS. Bennett \& Brachman's hospital infections, 5th ed. Philadelphia: Wolters Kluwer Health/Lippincott Williams \& Wilkins; 2007. p. 832. xiv.

18. Gordon RJ, Lowy FD. Bacterial infections in drug users. N Engl J Med 2005;353: 1945-54.

19. Leung DY. Infection in atopic dermatitis. Curr Opin Pediatr 2003;15:399-404.

20. Myers D, Baer WC, Choi SY. The changing problem of overcrowded housing. J Am Plann Assoc 1996;62:66-84.

21. McEwen BS. Protective and damaging effects of stress mediators. N Engl J Med 1998;338:171-9.

22. Elesh D, Lefcowitz MJ. The effects of the New Jersey-Pennsylvania Negative Income Tax Experiment on health and health care utilization. J Health Soc Behav 1977; 18:391-405.

23. Muennig P, Schweinhart L, Montie J, Neidell M. Effects of a prekindergarten educational intervention on adult health: 37-year follow-up results of a randomized controlled trial. Am J Public Health 2009;99:1431-7.

24. Muennig P, Johnson G, Wilde ET. The effect of small class sizes on mortality through age 29 Years: evidence from a multicenter randomized controlled trial. Am J Epidemiol 2011;173:1468-74.

25. Muennig P, Robertson D, Johnson G, Campbell F, Pungello EP, Neidell M. The effect of an early education program on adult health: the Carolina Abecedarian Project randomized controlled trial. Am J Public Health 2011;101:512-6.

26. Lawrence FK, Jeffrey RK, Jeffrey BL. Moving to opportunity in Boston: early results of a randomized mobility experiment. Q J Econ 2001;116:607.

27. Leventhal T, Brooks-Gunn J. Moving to opportunity: an experimental study of neighborhood effects on mental health. Am J Public Health 2003;93:1576-82.

28. Lagarde M, Haines A, Palmer N. The impact of conditional cash transfers on health outcomes and use of health services in low and middle income countries. Cochrane Database Syst Rev 2009;(4):CD008137.

29. Ozer EJ, Fernald LC, Manley JG, Gertler PJ. Effects of a conditional cash transfer program on children's behavior problems. Pediatrics 2009;123:e630-7.

30. Attanasio O, Meghir C, Schady N. Mexico's conditional cash transfer programme. Lancet 2010;375:980. author reply 1.

31. Barber SL. Mexico's conditional cash transfer programme increases cesarean section rates among the rural poor. Eur J Public Health 2010;20:383-8.

32. Lim SS, Dandona L, Hoisington JA, James SL, Hogan MC, Gakidou E. India's Janani Suraksha Yojana, a conditional cash transfer programme to increase births in health facilities: an impact evaluation. Lancet 2010;375:2009-23.

33. Adato M, Roopnaraine T, Becker E. Understanding use of health services in conditional cash transfer programs: insights from qualitative research in Latin America and Turkey. Soc Sci Med 2011;72:1921-9.

34. Paes-Sousa R, Santos LM, Miazaki ES. Effects of a conditional cash transfer programme on child nutrition in Brazil. Bull World Health Organ 2011;89:496-503. 